\title{
Physicochemical influences on electrohydrodynamic transport in compressible packed beds of colloidal boehmite particles
}

\author{
Bastian Schäfer*, Hermann Nirschl \\ Institut für Mechanische Verfahrenstechnik und Mechanik, Universität Karlsruhe (TH), Straße am Forum 8, 76131 Karlsruhe, Germany
}

Received 31 July 2007; accepted 2 October 2007

Available online 7 November 2007

\begin{abstract}
Production and processing of colloidal particles require a deeper understanding of the surface charge of particles and the interaction of mass and charge transport in packed beds. The assessment of fundamental parameters is rather complex due to the additional influence of the particle charge on the structure of a packed bed. The combination of different measurement techniques (streaming potential and electroosmosis) allows for separating the effects, based on the postulation of a new method to quantify the ratio of surface conductance to liquid conductance. The purpose of this paper is to investigate the influence of $\mathrm{pH}$ value and compression on the electrohydrodynamic transport parameters.
\end{abstract}

(C) 2007 Elsevier Inc. All rights reserved.

Keywords: Electrohydrodynamic transport; Electrokinetics; Electrostatics; Permeability; Colloidal particles

\section{Introduction}

In a packed bed single colloidal particles are randomly and densely distributed between two membranes while liquid flows through the porous structure. Understanding the influences of physicochemistry on the mass and charge transport in packed beds is important for industrial applications: Colloidal particles have promising applications, but the poor permeability of filter cakes limits mechanical separation and hence production and processing [1]. The consolidation of green bodies is essential for realizing transparent nanoceramics. Nanoporous packed beds could also be used in fixed-bed catalyst reactors and electroosmotic micropumps. Porous ceramic bodies are used as a barrier between the anode and the cathode in lithium ion batteries.

The problem of the very low permeability can be overcome by influencing the agglomerate structure of the particles or by utilizing the interaction of hydraulic and electric transport. Therefore the focus of this paper is to understand the combination of electrohydrodynamic transport phenomena and elec-

\footnotetext{
* Corresponding author. Fax: +497216082403 .

E-mail addresses: bastian.schaefer@mvm.uka.de (B. Schäfer), hermann.nirschl@mvm.uka.de (H. Nirschl).
}

trostatic effects on the packed-bed structure [2] and to separate them from each other.

In this study, the influence of the $\mathrm{pH}$ value and compression on the transport through a nanoporous packed bed is investigated. The packed beds are formed by filtration and compression of colloidal boehmite suspensions. A new method is developed for combining different measurement techniques in order to separate the interrelated influences. The method allows for determining the surface charge and the electrohydrodynamic transport in packed beds. The experimental conditions are well in the range of the thin electrochemical double layer (EDL).

\section{Theoretical background}

Electrohydrodynamic transport phenomena in nanoporous packed beds are closely related to the particle charge and its influence on the pore structure of packed beds. The EDL on ceramic particles in aqueous suspensions originates from dissociation reactions on the particle surface atoms (denoted by $\mathrm{S}$ ) $[3,4]$. The equilibrium of the dissociation depends on the concentration of hydroxide ions:

$$
\begin{aligned}
& \mathrm{SOH} \leftrightharpoons \mathrm{SO}^{-}+\mathrm{H}^{+}, \\
& \mathrm{SOH}+\mathrm{H}_{2} \mathrm{O} \leftrightharpoons \mathrm{SOH}_{2}^{+}+\mathrm{OH}^{-} .
\end{aligned}
$$


The material-specific $\mathrm{pH}$ value for which the surface charge equals zero is called isoelectric point (IEP). Below the IEP the particle charge is positive and above the IEP it becomes negative. The particle is surrounded by a diffuse cloud of counterions, which are attracted to the particle by electrostatic forces. The exponential decay length of the ion concentration, the Debye length $\kappa^{-1}$, is a function of the ionic strength $\tilde{I}$ with the Faraday constant $F$, the relative permittivity of the liquid $\varepsilon_{\mathrm{L}}$, the vacuum permittivity $\varepsilon_{0}$, the gas constant $R$, and the temperature $T[3,4]$ :

$\kappa^{-1}=\left(\frac{2 F^{2} \tilde{I}}{\varepsilon_{\mathrm{L}} \varepsilon_{0} R T}\right)^{-(1 / 2)}$.

In our study, at $\tilde{I}=0.01 \mathrm{M}$ and $T=288 \mathrm{~K}$, the Debye length is approximately $3 \mathrm{~nm}$. Pragmatically, the EDL is subdivided into the immobile Stern layer (SL) and the surrounding diffuse layer (DL). As the surface charge density is difficult to measure, the EDL is often characterized by the zeta potential $\zeta$, which is more relevant for practice and easier to determine, e.g., by analyzing the electrokinetic effects $[3,5]$. The latter are described by cross coefficients in the electrohydrodynamic transport equations, which relate the volumetric flow $\dot{V}$ and electric current $I$ to the difference of the pressure $p$ and the electric potential $\Psi_{\text {Ext }}$ :

$\dot{V}=C_{11} \Delta(-p)+C_{12} \Delta\left(-\Psi_{\text {Ext }}\right)$,

$I=C_{21} \Delta(-p)+C_{22} \Delta\left(-\Psi_{\text {Ext }}\right)$.

$C_{11}$ to $C_{22}$ are material-specific coefficients. $C_{12}$ equals $C_{21}$ according to Onsager's relation [6].

- $C_{11}$ is related to Darcy's law, which describes the flow rate $\dot{V}$ of a liquid with the viscosity $\eta_{\mathrm{L}}$ driven by the pressure difference $\Delta(-p)$ depending on the cross-sectional area $A_{\mathrm{PB}}$ and the thickness $L_{\mathrm{PB}}$ of a packed bed [7]:

$\dot{V}=\frac{K_{\mathrm{hydr}} A_{\mathrm{PB}}}{\eta_{\mathrm{L}} L_{\mathrm{PB}}} \Delta(-p)$.

Several models for the permeability $K_{\text {hydr }}$ have been proposed for macroporous packed beds with a homogeneous pore size, e.g., by Carman [8]. Briefly, an increase of porosity and particle size leads to a higher permeability. In the case of a compressible packed bed, an increase of compression leads to a decrease of porosity [9]. As the liquid permeates the structure, the liquid pressure is reduced due to friction and transmitted to the solid structure. Consequently, the compression has a maximum and the porosity has a minimum at the downstream membrane [9]. In this study, the effect of an inhomogeneous compression can be neglected as the packed bed is consolidated evenly by a plunger. For nanoporous packed beds, the porosity and the pore size distribution depend largely on the electrostatic effect, i.e., the influence of the particle charge on the agglomerate structure [2]. Agglomeration is determined by the equilibrium of electrostatic repulsion, van der Waals attraction and Born's repulsion between particles as described by the DLVO theory. It can be investigated by small-angle X-ray scattering $[3,4,10]$. Suspensions with a low particle charge tend to agglomerate, leading to a loosely structured packed bed with an inhomogeneous pore size distribution. The large pores between the agglomerates are accountable for the high permeability. In contrast, a stable suspension with a high zeta potential leads to a dense structure with a homogeneous pore size distribution and a low permeability [2].

- $C_{12}$ describes the electroosmotic mass transport, which is driven by an external electric potential $\Psi_{\text {Ext }}$. The counterions in the DL of the particles are accelerated and drag the adjacent water molecules along. Smoluchowski's assumption of a thin double layer, or more precisely $\kappa a \gg 1$ with the reciprocal Debye length $\kappa$ and the radius of curvature $a$, is fulfilled here. In this case, the electroosmotic flow $\dot{V}_{\mathrm{EO}}$ in a hypothetical cylindrical capillary depends on the zeta potential and the cross-sectional area $A_{\text {Cap }}[3,5]$ :

$\dot{V}_{\mathrm{EO}}=-\frac{\varepsilon_{\mathrm{L}} \varepsilon_{0} \zeta}{\eta_{\mathrm{L}}} A_{\mathrm{Cap}} \cdot \Delta\left(-\Psi_{\mathrm{Ext}}\right)$.

The electric potential $\Delta\left(-\Psi_{\text {Ext }}\right)$ is the quotient of the externally applied voltage $U_{\text {Ext }}$ and the length of the capillary $L_{\mathrm{Cap}}$. Experiments showed that the complex pore geometry of a packed bed with the cross-sectional area $A_{\mathrm{PB}}$, the thickness $L_{\mathrm{PB}}$, and the porosity $\phi_{\mathrm{L}}$ can be accounted for by substituting $A_{\mathrm{Cap}} / L_{\mathrm{Cap}}$ with $A_{\mathrm{PB}} / L_{\mathrm{PB}} \cdot \phi_{\mathrm{L}}^{2.5}[5,11]$ :

$\dot{V}_{\mathrm{EO}}=-\frac{\varepsilon_{\mathrm{L}} \varepsilon_{0} \zeta}{\eta_{\mathrm{L}}} \frac{A_{\mathrm{Cap}}}{L_{\mathrm{Cap}}} U_{\mathrm{Ext}}=-\frac{\varepsilon_{\mathrm{L}} \varepsilon_{0} \zeta}{\eta_{\mathrm{L}}} \frac{A_{\mathrm{PB}}}{L_{\mathrm{PB}}} \phi_{\mathrm{L}}^{2.5} U_{\mathrm{Ext}}$.

- $C_{21}$ specifies the streaming current $I_{\mathrm{Str}}$, which is the charge transport generated when counterions are dragged from the DL by a pressure-driven liquid flow. The streaming current can be measured if the inlet face of the structure is connected to the outlet face with a low-resistance ampere meter (short-circuit conditions) [5]:

$I_{\mathrm{Str}}=-\frac{\varepsilon_{\mathrm{L}} \varepsilon_{0} \zeta}{\eta_{\mathrm{L}}} \frac{A_{\mathrm{Cap}}}{L_{\mathrm{Cap}}} \Delta(-p)=-\frac{\varepsilon_{\mathrm{L}} \varepsilon_{0} \zeta}{\eta_{\mathrm{L}}} \frac{A_{\mathrm{PB}}}{L_{\mathrm{PB}}} \phi_{\mathrm{L}}^{2.5} \Delta(-p)$.

- $C_{22}$ is the electric conductance of the porous structure. The conductivity $K$ comprises liquid conduction $K_{\mathrm{L}}$ (ion movement in the electrolyte solution in the pores) and surface conduction $K_{\text {Surf }}$ (movement of excess ions in the DL and SL). Surface conductance causes a "short-circuiting" inside the packed bed, which must not be neglected for a correct interpretation of electrohydrodynamics. For a cylindrical capillary with the radius $r_{\text {Cap }}$, geometrical considerations lead to

$K=K_{\mathrm{L}}+2 K_{\text {Surf }} / r_{\text {Cap }}=K_{\mathrm{L}}(1+2 \mathrm{Du})$

with the Dukhin number Du $[5,12]$ :

$\mathrm{Du}=K_{\text {Surf }} /\left(K_{\mathrm{L}} r_{\text {Cap }}\right)=\frac{1}{2}\left(\frac{K}{K_{\mathrm{L}}}-1\right)$.

The total conductivity of the sample is calculated as $K=$ $(L / A) / R$, with the geometry factor $L / A$ and the electric resistance $R$. For a nondeformable structure the geometry factor can be determined from the resistance $R^{\infty}$ when the structure is filled with high ionic strength electrolyte solution with the conductivity $K_{\mathrm{L}}^{\infty}$, so that surface conduction is negligible:

$L / A=K_{\mathrm{L}}^{\infty} R^{\infty}$. 
However, the technique is not applicable to packed beds since the increase of ionic strength influences the packed bed's structure significantly. We propose to derive the Dukhin number from Eq. (8) with the zeta potential from Eq. (6) and the ratio of the electroosmotic flow to the electric current $I_{\text {Ext }}$ as given by [5]

$$
\begin{aligned}
& \frac{\dot{V}_{\mathrm{EO}}}{I_{\mathrm{Ext}}}=-\frac{\varepsilon_{\mathrm{L}} \varepsilon_{0} \zeta}{\eta_{\mathrm{L}}} \frac{R_{\mathrm{PB}}}{K_{\mathrm{L}}^{\infty} R^{\infty}}, \\
& \mathrm{Du}=-\frac{1}{2}\left(\frac{\varepsilon_{\mathrm{L}} \varepsilon_{0} \zeta}{\eta K_{\mathrm{L}}\left(\dot{V}_{\mathrm{EO}} / I_{\mathrm{Ext}}\right)}+1\right) .
\end{aligned}
$$

Our approach is analogous to a method introduced by [13], but is not limited to straight capillaries.

- If counterions are dragged from the DL by a pressuredriven liquid flow and the faces of the packed bed are not short-circuited, the streaming current must be balanced. Hence, the streaming potential $U_{\text {Str }}$ is built up, which drives counterions back against the pressure-driven flow. $U_{\text {Str }}$ is measured between an upstream and a downstream electrode and can be used to determine $\zeta$ if surface conduction is considered. Surface conduction reduces the potential required to balance the streaming current $[3,5]$ :

$U_{\mathrm{Str}}=\frac{\varepsilon_{\mathrm{L}} \varepsilon_{0} \zeta}{\eta_{\mathrm{L}}} \frac{R_{\mathrm{PB}}}{K_{\mathrm{L}}^{\infty} R_{\mathrm{PB}}^{\infty}} \Delta(-p)$.

- The counterions driven against the flow by the streaming potential induce an electroosmotic backflow, which retards the permeation. This apparent rise of the fluid viscosity, the so-called electroviscous retardation, can be calculated if the electric current $I$ in Eq. (3) is set to zero [14]:

$\dot{V}=\left(C_{11}-\frac{C_{12} C_{21}}{C_{22}}\right) \Delta(-p)$.

We define the electroviscosity ratio EVR as the ratio between the apparent viscosity $\eta_{\text {App }}$ and the real viscosity of the permeate $\eta_{\mathrm{L}}$ :

$\mathrm{EVR}=\frac{\eta_{\mathrm{App}}-\eta_{\mathrm{L}}}{\eta_{\mathrm{L}}}=\frac{C_{12} C_{21}}{C_{11} C_{22}-C_{12}^{2}}$.

The EVR allows for quantifying the electroviscous retardation.

\section{Experiments}

Packed beds are formed in the cylindrical electro-compression permeability cell (ECP-Cell) by filtration of $200 \mathrm{~g}$ suspension. The packed bed is then compressed by a plunger and permeated by a voltage or a pressure difference. The two-sided filtration between two nylon membranes (Ultipor from Pall, USA, with a nominal pore size of $0.1 \mu \mathrm{m}$ ) in the vertical position of the ECP-Cell and the low thickness-to-diameter ratio ensure a constant compression and thus homogeneous structure of the packed bed. All parts of the ECP-Cell except for the electrodes are nonconducting. After the filtrate flow abates, the ECP-Cell is turned to the horizontal position, which is shown in Fig. 1.

The packed bed is cooled by electrolyte solution circulated through heat exchangers. The solution has the same ionic

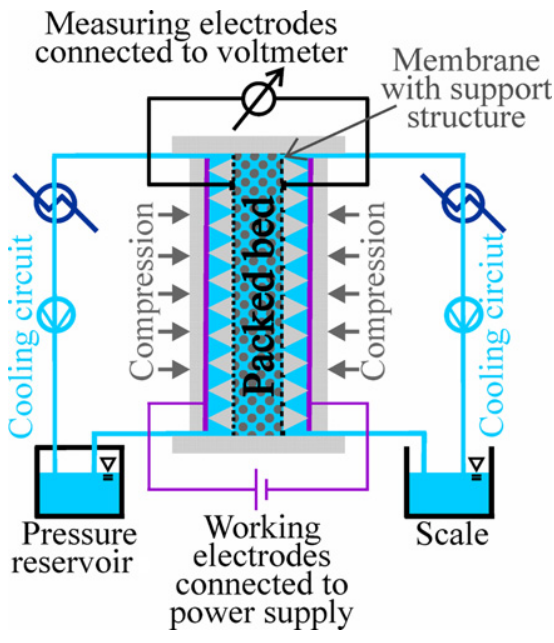

Fig. 1. Electro-compression permeability cell in horizontal position.

strength and $\mathrm{pH}$ value as the suspension. After reaching a constant temperature of $288 \mathrm{~K}$, the circulation stops and the packed bed is permeated by the electrolyte solution, driven by a pressure difference or by an electric field. The scale displays the accumulation of mass on the downstream side.

The streaming potential evoked by a pressure-driven permeation is measured with platinum electrodes (measuring electrodes). Alternatively, an electric field applied via the working electrodes excites electroosmotic permeation. The iridium oxide coating of the titanium electrodes prevents oxidation. If the electric field is reversed every $30 \mathrm{~s}$, the formation of electrolytic gas is prevented because hydrogen formed at the cathode reacts with oxygen from the previous cycle. The left working electrode is defined as anode, the right one as cathode. A negative electric field means that voltage and flow are reversed. The effective voltage at the packed bed is monitored with the measuring electrodes because the voltage is reduced due to the electric resistance of the electrolyte solution in the cooling circuit.

The suspensions are prepared by dispersing $25 \mathrm{~g}$ boehmite particles (Disperal 40 from Sasol, Germany, with an average particle size of $350 \mathrm{~nm}$ and a standard deviation of $60.7 \mathrm{~nm}$ ) in $225 \mathrm{~g}$ electrolyte solution with a propeller stirrer for $15 \mathrm{~min}$. The electrolyte solution has an ionic strength of $0.01 \mathrm{M}$ and a $\mathrm{pH}$ value of 4 , adjusted with $\mathrm{KNO}_{3}$ and $\mathrm{HNO}_{3}$. While for most particles an elaborate method for dispersing is required [15-17], boehmite particles are easily dispersed in acidic solutions, which was verified with a Coulter N4 Plus. Subsequently, the suspension is flocculated by injecting different amounts of $1 \mathrm{M} \mathrm{KOH}$ into the suspension and stirred for further $30 \mathrm{~min}$ to homogenize the suspension. The background electrolyte $\mathrm{KNO}_{3}$ guarantees the well definition of the surface charge [5]. The zeta potential of the suspensions was determined with an AcoustoSizer II (see Fig. 5). Boehmite particles are common as filler in synthetics and raw material for the aluminum oxide industry. Boehmite is dissolvable in aqueous solutions, especially at high and low $\mathrm{pH}$ values. However, at temperatures below $50{ }^{\circ} \mathrm{C}$ the kinetics are so slow that the solubility can be neglected [18]. 


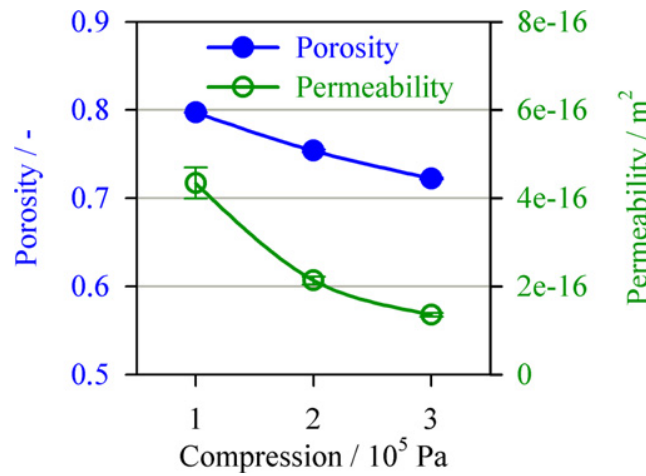

Fig. 2. Porosity and permeability depending on compression for packed beds with a $\mathrm{pH}$ value of 10.9 and an ionic strength of $0.01 \mathrm{M}$.

\section{Results}

For the interpretation of the measurements a homogeneous compression of the packed bed and thus a homogeneous porosity are assumed because the compression from the plunger is significantly higher than the pressure drop of the permeate. The porosity was calculated from the thickness of the packed bed $L_{\mathrm{PB}}$ and the mass of the particles $m_{\mathrm{S}}$ :

$\phi_{\mathrm{L}}=\frac{\left(L_{\mathrm{PB}} A_{\mathrm{PB}}-\left(m_{\mathrm{S}} / \rho_{\mathrm{S}}\right)\right)}{L_{\mathrm{PB}} A_{\mathrm{PB}}}$.

In all figures the error bars represent the standard deviation.

A higher compression leads to a lower porosity (see Fig. 2). The small reduction of the porosity (from 0.8 to 0.72 ) goes along with a strong decrease of the permeability, since mainly the large pores between the agglomerates are affected. These are accountable for the major part of the fluid transport $[2,19]$.

Porosity also changes with a variation of $\mathrm{pH}$ value (see Fig. 3) due to the agglomeration of the particles when the $\mathrm{pH}$ value approaches the IEP at $\mathrm{pH} 9.5$ (compare Fig. 5). Beyond the IEP, the porosity remains high because of the sample preparation process: The particles can only be dispersed at a low $\mathrm{pH}$ value. In order to obtain a high $\mathrm{pH}$ value, the IEP must be passed. The agglomerates formed at the IEP are not disagglomerated thereafter because the shear gradients caused by the stirrer are too small. Again, the increase of the porosity causes an increased permeability at the IEP and beyond it.

The permeability is identical for points of the same porosity when comparing Fig. 2 and Fig. 3. A packed bed from an agglomerated suspension at high compression and a packed bed from an unagglomerated suspension at low compression have the same permeability if the porosity is identical. Accordingly, the change of permeability on variation of the $\mathrm{pH}$ value is attributed to a change of porosity and there is no further effect of the agglomerate structure of the packed bed on the permeability.

For thin double layers $(\kappa a \gg 1)$ the zeta potential is not a function of the compression (see Fig. 4), as it is not affected by the reduction of the pore size because the EDL of neighboring particles do not overlap. $\zeta_{\dot{V}_{\mathrm{EO}} / \Delta(-\psi)}$ is calculated with Eq. (6) and $\zeta_{U / \Delta(-P)}$ with Eq. (13). $R_{\mathrm{PB}} /\left(K_{\mathrm{L}}^{\infty} R_{\mathrm{PB}}^{\infty}\right)$ is determined from the Dukhin number (see below).

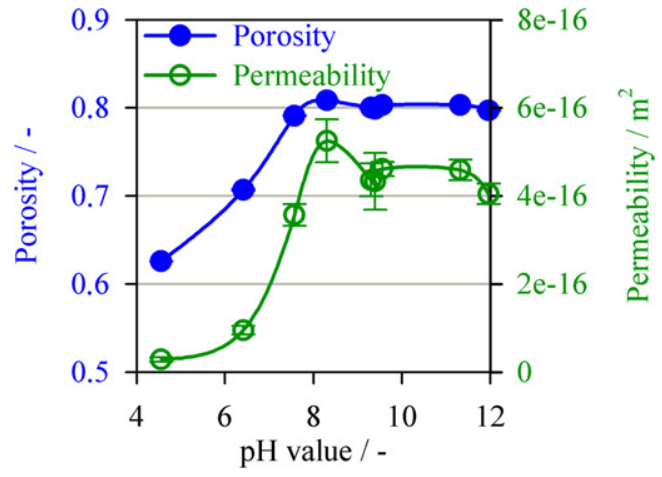

Fig. 3. Porosity and permeability depending on $\mathrm{pH}$ value for packed beds with a compression of $100 \mathrm{kPa}$ and an ionic strength of $0.01 \mathrm{M}$.

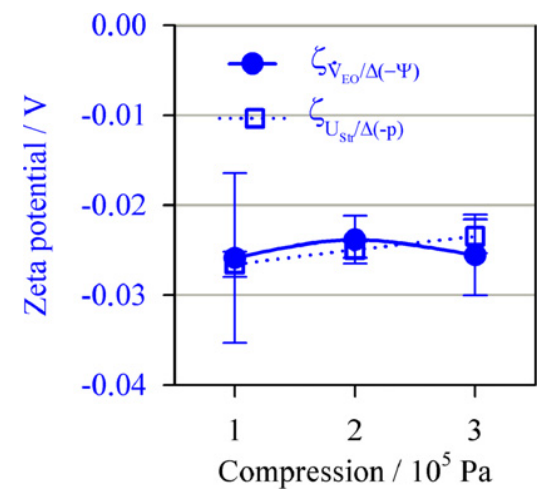

Fig. 4. Zeta potential $\zeta$ depending on compression for packed beds with a $\mathrm{pH}$ value of 10.9 and an ionic strength of $0.01 \mathrm{M} . \zeta_{\dot{V}_{\mathrm{EO}} / \Delta(-\psi)}$ is calculated from the electroosmotic flow and $\zeta_{U_{\mathrm{Str}} / \Delta(-P)}$ is determined from the streaming potential.

$\zeta_{U_{\text {Str }} / \Delta(-p)}$ is consistent with zeta potential calculated from the electroosmotic flow $\left(\zeta_{\dot{V}_{\mathrm{EO}} / \Delta(-\psi)}\right)$ and with electroacoustic measurements (see Fig. 3). The latter were determined with an AcoustoSizer II in a suspension of $10 \mathrm{wt} \%$ and an ionic strength of $0.01 \mathrm{M}$ assuming the fulfillment of the Smoluchowski dynamic electroacoustic limit $\left(\zeta_{\mathrm{EA}, \mathrm{Smol}}\right)$ and with the O'Brien algorithm ( $\left.\zeta_{\mathrm{EA}, \mathrm{O}^{\prime} \text { Brien }}\right)$. The discrepancy between $\zeta_{\dot{V}_{\mathrm{EO}} / \Delta(-\psi)}$, $\zeta_{U / \Delta(-P)}$ and the AcoustoSizer measurements is in the same order of magnitude as the discrepancy in between the AcoustoSizer measurements. This was expected, as Fig. 4 shows that the solid concentration has no influence on $\zeta$ in the limit of thin double layers. Therefore we can conclude that our measurement techniques and our approach to the Dukhin number are valuable.

For the experiments at a $\mathrm{pH}$ value of 10.9 , the ratio of the electroosmotic flow to the externally applied electric current $\dot{V}_{\mathrm{EO}} / I_{\mathrm{Ext}}$ is $6 \times 10^{-8} \mathrm{~m}^{3} / \mathrm{A}$. This corresponds to $\dot{V} / I \cdot N_{\mathrm{A}}$. $\rho_{\mathrm{L}} / M=2 \times 10^{21}$ water molecules per Coulomb or $\dot{V} / I \cdot F$. $\rho_{\mathrm{L}} / M=318$ water molecules dragged by each counterion with the Avogadro constant $N_{\mathrm{A}}$, the Faraday constant $F$, and the molar mass $M . \dot{V}_{\mathrm{EO}} / I_{\mathrm{Ext}}$ does not depend on the compression and thus pore size as the acceleration in the EDL causes the plug profile of electroosmotic flow, so that the velocity is independent of the pore size [3]. $\dot{V}_{\mathrm{EO}} / I_{\mathrm{Ext}}$ has the same tendency as $\zeta$ when the $\mathrm{pH}$ value is varied. 


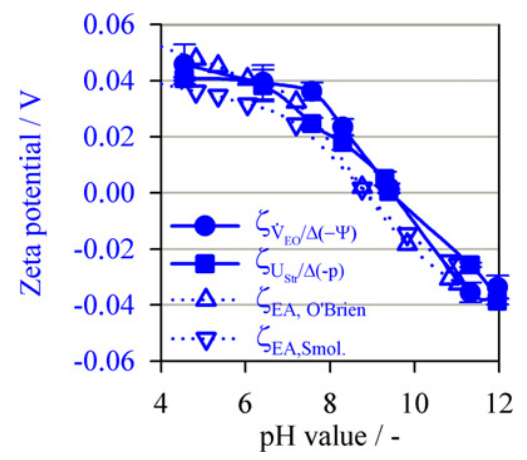

Fig. 5. Zeta potential $\zeta$ depending on $\mathrm{pH}$ value for packed beds with a compression of $100 \mathrm{kPa}$ and an ionic strength of $0.01 \mathrm{M}$. The symbols are explained in the text.

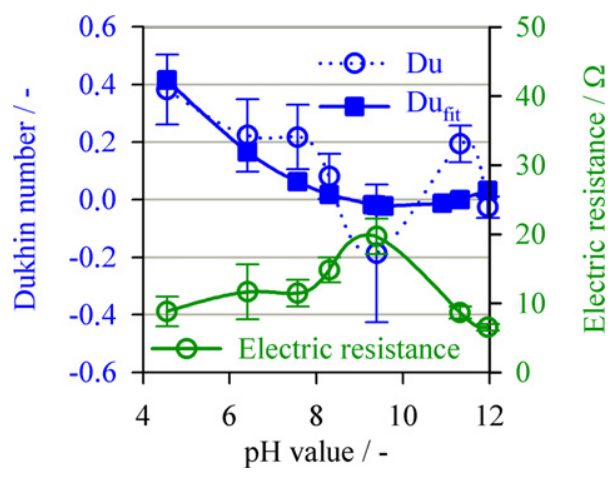

Fig. 6. Dukhin number $(\mathrm{Du})$, fit of the Dukhin number $\left(\mathrm{Du}_{\mathrm{fit}}\right)$, and electric resistance depending on the $\mathrm{pH}$ value for packed beds with a compression of $100 \mathrm{kPa}$ and an ionic strength of $0.01 \mathrm{M}$.

The Dukhin number (Du) is calculated by using Eq. (12) with $\zeta_{\dot{V}_{\mathrm{EO}} / \Delta(-\psi)}$, from Fig. 5 and the measured ratio of the electroosmotic flow to the externally applied electric current. For the experiments at a $\mathrm{pH}$ value of 10.9 , a Dukhin number of 0.23 was found, which is independent of the compression. This is reasonable for thin double layers as the reduction of pore size does not influence the surface conduction. The compression is expected to influence the Dukhin number only when the thin double layer approximation is not valid.

In contrast, the influence of the $\mathrm{pH}$ value on the Dukhin number is fitted with a quadratic function (see Fig. 6). The minimum of the Dukhin number at the IEP is caused by the low zeta potential: The weak EDL does not cause an excess surface conduction, which can also be seen from the maximum of the electric resistance. The reduced volume available for charge transport may be the reason for the negative value of Du. But as both $\zeta$ and $\dot{V}_{\mathrm{EO}}$ in Eq. (12) are close to zero at the IEP, the absolute value and the negative sign are rather uncertain.

The electroviscosity ratio EVR increases with increasing compression (see Table 1) because a larger part of the flow takes place inside of the EDL when the pores get smaller. The electroviscosity ratio is zero at the IEP (see Fig. 7), where no streaming potential is built up and no electroosmotic backflow evolves. Beyond the IEP, EVR is smaller than below the IEP because of the increased porosity due to agglomeration. But altogether, the increase of the apparent viscosity is negligible in
Table 1

Electroviscosity ratio depending on compression for packed beds with an ionic strength of $I=0.01 \mathrm{M}$

\begin{tabular}{lll}
\hline $\begin{array}{l}\mathrm{pH} \text { value } \\
(-)\end{array}$ & $\begin{array}{l}\text { Compression } \\
(\mathrm{Pa})\end{array}$ & $\begin{array}{l}\text { Electroviscosity } \\
\text { ratio }(-)\end{array}$ \\
\hline 10.9 & $1 \times 10^{5}$ & $9.86 \mathrm{e}-6$ \\
10.9 & $2 \times 10^{5}$ & $1.26 \mathrm{e}-5$ \\
10.9 & $3 \times 10^{5}$ & $1.92 \mathrm{e}-5$ \\
\hline
\end{tabular}

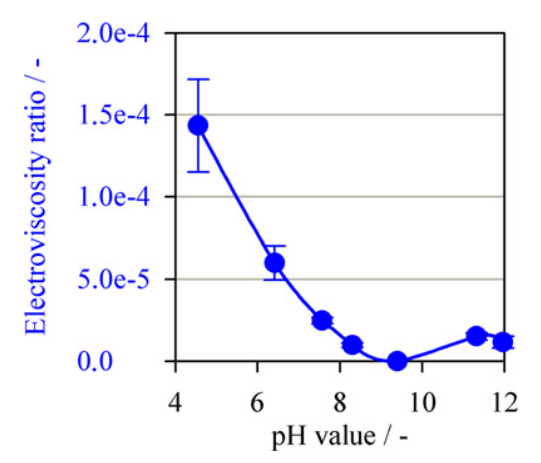

Fig. 7. Electroviscosity ratio depending on $\mathrm{pH}$ value for packed beds with a compression of $100 \mathrm{kPa}$ and an ionic strength of $0.01 \mathrm{M}$.

the thin double layer limit (i.e., $\kappa a \gg 1$ ). This corresponds to the finding that the permeability is identical for points of the same porosity when comparing Fig. 2 and Fig. 3. Accordingly, the change of permeability on variation of the $\mathrm{pH}$ value is attributed to a change of porosity, not to the electroviscosity.

\section{Summary}

The interrelation of the influence of particle charge on the structure of a packed bed with the interactions of charge and mass transport complicates the understanding of electrohydrodynamic transport phenomena. A combination of different measurement techniques (streaming potential and electroosmosis) allows for separating the effects, based on the postulation of a new method to quantify the ratio of surface conductance to liquid conductance. The influence of $\mathrm{pH}$ value and compression is in the focus of this paper.

The zeta potential of the particles in the packed bed depending on $\mathrm{pH}$ value is consistent with the zeta potential of suspensions measured with an AcoustoSizer II. $\zeta$ does not depend on the compression of the packed bed in the case of thin double layers (i.e., $\kappa a \gg 1$ ). Also the ratio of the electroosmotic flow to the electric current is independent of the compression. The ratio of surface conductance to liquid conductance has a minimum at the isoelectric point. The electroviscous retardation of a hydraulic permeation is negligible and the change of permeability on variation of the $\mathrm{pH}$ value is attributed to the change of the porosity.

Our approach offers the possibility of accessing the electrohydrodynamic transport properties of nanoporous materials. Future studies will include the influence of ionic strength and particle size, so that a basis for industrial applications will be established. 


\section{Acknowledgment}

We thank the German Science Foundation (DFG) for funding within the priority program SPP 1164.

\section{References}

[1] H. Nirschl, R. Wengeler, B. Schäfer, Filtration 7 (2007) 67.

[2] B. Schäfer, H. Nirschl, Chem. Eng. Technol. 28 (2005) 862.

[3] J. Lyklema (Ed.), Fundamentals of Interface and Colloid Science II, Academic Press, London, 1995.

[4] R.J. Hunter, Introduction to Modern Colloid Science, Oxford Univ. Press, Oxford, 1993.

[5] A.V. Delgado, et al., J. Colloid Interface Sci. 309 (2007) 194.

[6] A.T. Yeung, J.K. Mitchell, Geotechnique 43 (1993) 121.

[7] H. Darcy, Les Fontaines Publiques de la Ville de Dijon, Dalmont, Paris, 1856.
[8] P.C. Carman, Trans. Inst. Chem. Eng. 15 (1937) 150.

[9] F.M. Tiller, C.S. Yeh, W.F. Leu, Sep. Sci. Technol. 22 (1987) 1037.

[10] R. Wengeler, F. Wolf, N. Dingenouts, H. Nirschl, Langmuir 23 (2007) 4148.

[11] G.J. Biefer, S.G. Mason, Trans. Faraday Soc. 55 (1959) 1234.

[12] R.J. Hunter, Adv. Colloid Interface Sci. 100-102 (2003) 153.

[13] R. Zimmermann, et al., Langmuir 20 (2004) 2369.

[14] N.P.R. Andersen, M.L. Agerbaek, K. Keiding, Colloids Surf. A 213 (2003) 27.

[15] R. Wengeler, H. Nirschl, J. Colloid Interface Sci. 306 (2007) 262.

[16] F. Müller, W. Peukert, R. Polke, F. Stenger, Int. J. Miner. Process. 74 (Suppl. 1) (2004) 31.

[17] K.A. Kusters, S.E. Pratsinis, S.G. Thoma, G.M. Smith, Chem. Eng. Sci. 48 (1993) 4119.

[18] D. Panias, P. Asimidis, I. Paspaliaris, Hydrometallurgy 59 (2001) 15.

[19] D. Antelmi, B. Cabane, M. Meireles, P. Aimar, Langmuir 17 (2001) 7137. 